OPEN ACCESS

Edited by:

Francesco Sofi,

Università degli Studi di Firenze, Italy

Reviewed by:

Holger Steinbrenner,

Friedrich Schiller University

Jena, Germany

Tommaso Filippini,

University of Modena and Reggio

Emilia, Italy

*Correspondence:

Liegang Liu

Igliu@mails.tjmu.edu.cn

Cheng LuO

1343941286@qq.com

Specialty section: This article was submitted to

Nutritional Epidemiology,

a section of the journal

Frontiers in Nutrition

Received: 05 October 2021 Accepted: 08 November 2021 Published: 07 December 2021

Citation:

Zhou L, Liang X, Xie M, Yin J, Huang Y, Li X, Shan Z, Chen L, Zhang Y, Luo C and Liu L (2021) A Functional Variant

in SEPP1 Interacts With Plasma

Selenium Concentrations on 3-Year Lipid Changes: A Prospective Cohort Study. Front. Nutr. 8:789577. doi: 10.3389/fnut.2021.789577

\section{A Functional Variant in SEPP1 Interacts With Plasma Selenium Concentrations on 3-Year Lipid Changes: A Prospective Cohort Study}

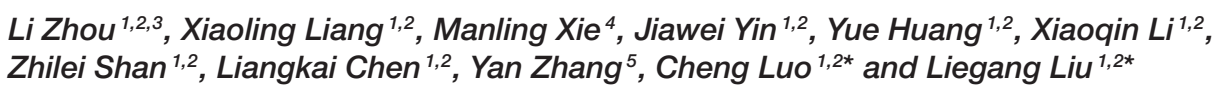

${ }^{1}$ Department of Nutrition and Food Hygiene, Hubei Key Laboratory of Food Nutrition and Safety, School of Public Health, Tongji Medical College, Huazhong University of Science and Technology, Wuhan, China, ${ }^{2}$ Ministry of Education (MOE) Key Lab of Environment and Health, School of Public Health, Tongji Medical College, Huazhong University of Science and Technology, Wuhan, China, ${ }^{3}$ Academy of Nutrition and Health, Wuhan University of Science and Technology, Wuhan, China, ${ }^{4}$ Department of Neurology, Mayo Clinic, Rochester, MN, United States, ${ }^{5}$ The Hubei Provincial Key Laboratory of Yeast Function, Yichang, China

Background: Excess selenium has been related with adverse lipid levels in previous epidemiological studies. Meanwhile, a functional variant in SEPP1 (encodes selenoprotein P), namely rs7579, has been suggested to modulate lipid metabolism. However, the interactions between selenium status and rs7579 polymorphism on lipid changes remain unclear.

Objective: To examine whether the associations between plasma selenium and 3-year lipid changes is modified by rs7579 polymorphism.

Methods: A prospective cohort study was conducted among 1,621 individuals to examine the associations between baseline plasma selenium and 3-year lipid changes, as well as the interactions between plasma selenium and rs7579 polymorphism on lipid changes.

Results: The median (interquartile range) concentration of plasma selenium was 91.68 (81.55-104.92) $\mu \mathrm{g} / \mathrm{L}$. Higher plasma selenium was associated with adverse 3-year lipid changes. Comparing the highest to the lowest quartiles of plasma selenium concentrations, 3-year lipid changes were elevated by $8.25 \%$ (95\% Cl: 1.54-14.96\%) for triglycerides $(P=0.016), 5.88 \%(3.13-8.63 \%)$ for total cholesterol $(P<0.001), 7.37 \%$ (3.07-11.67\%) for low-density lipoprotein cholesterol $(P=0.0008), 6.44 \%(2.66-10.21 \%)$ for non-high-density lipoprotein cholesterol $(P=0.0009), 4.99 \%(0.62-9.36 \%)$ for total cholesterol/high-density lipoprotein cholesterol ratio $(P=0.025)$, and $7.00 \%(1.55-12.46 \%)$ for low-density lipoprotein cholesterol/high-density lipoprotein cholesterol ratio $(P=0.012)$. In analyses stratified by rs7579 genotypes, positive associations between plasma selenium concentrations and 3-year changes in triglycerides, TC, LDL-C, non-HDL-C, TC/HDL-C ratio, and LDL-C/HDL-C ratio were observed among CC 

genotype carriers, but negative associations between plasma selenium and TC/HDL-C
ratio, and LDL-C/HDL-C ratio were observed among $T$ T genotype carriers.

Conclusions: Our findings suggested that plasma selenium was associated with 3-year lipid changes differentially by rs7579 genotypes, and higher plasma selenium was associated with adverse lipid changes among rs7579 CC genotype carriers, but not among $T$ allele carriers.

Keywords: selenium, SEPP1, gene-environment interactions, lipid changes, cohort study

\section{INTRODUCTION}

Selenium is an essential trace element which plays an important role in the antioxidant process by incorporating into selenoproteins (1). Because of this property, selenium supplementation has been suggested to protect against oxidative stress. In recent years, selenium-containing supplements and selenium-rich foods have been widely used due to their potential benefits (2). However, several studies revealed that high selenium was associated with numerous metabolic disorders, including dyslipidemia (3-5). Most epidemiological studies examining the associations between selenium status and lipid levels are cross-sectional, while longitudinal evidence to show prospective associations is limited $(3,6-8)$.

Genetic factors are believed to play an important role in lipid metabolism $(9,10)$. A functional variant located in the $3^{\prime}$ untranslated regions of SEPP1, namely rs7579 polymorphism, has been suggested to modulate lipid response to Brazil nut supplementation, and Brazil nut is the richest natural source of selenium (11). SEPP1 encodes selenoprotein P (SELENOP), which mainly functions as a selenium transporter and delivers selenium from the liver to other tissues $(12,13)$. Moreover, findings from previous studies indicated that SELENOP played a crucial role in the antioxidant-mediated protection against colitis-associated cancer $(14,15)$. Several studies demonstrated that the rs7579 polymorphism affected the mRNA expression as well as plasma levels of SELENOP, and both the mRNA expression and plasma levels of SELENOP were significantly lower among rs7579 CC genotype than $\mathrm{T}$ allele carriers (16, 17). Meanwhile, previous findings showed that the changes in cholesterol levels were greater among rs7579 CC genotype than $\mathrm{T}$ allele carriers in response to Brazil nut supplementation (18). Based on the function of rs7579 polymorphism, it can be anticipated that the associations between plasma selenium and changes in lipid levels are more pronounced in rs7579 CC genotype than T allele carriers. However, to our knowledge, no study has examined the interaction between plasma selenium and rs7579 polymorphism on lipid changes.

Hence, the current study aimed to evaluate the associations between plasma selenium concentrations and changes in lipid

\footnotetext{
Abbreviations: BMI, body mass index; FASN, fatty acid synthase; HDL-C, high-density lipoprotein cholesterol; LDL-C, low-density lipoprotein cholesterol; NHANES III, the third National Health and Nutrition Examination Survey; SELENOP, selenoprotein P; SREBP1c, sterol regulatory element binding protein 1c; TC, total cholesterol; TJEZ, Tongji-Ezhou.
}

levels, and then to examine whether the associations differ across rs7579 genotypes.

\section{METHODS}

\section{Study Design and Subjects}

The current study was conducted based on the Tongji-Ezhou (TJEZ) cohort study, which is an ongoing prospective cohort study, with the aim of investigating the associations of lifestyle, dietary factors, and genetic factors with chronic diseases. Design of the TJEZ cohort study has been described elsewhere (5). Briefly, 5,533 residents in Ezhou aged above 20 years were recruited between 2013 and 2015. At baseline, all participants underwent comprehensive medical examinations, including semi-structured questionnaires, anthropometric measurements, and biochemical analyses (including lipid measurements). Also, fasting venous blood samples were collected from all participants at baseline enrollment. All blood samples were separated for plasma within $1 \mathrm{~h}$ and stored in $-80^{\circ} \mathrm{C}$ freezers until laboratory analysis. Additionally, genotyping of rs7579 was performed on 3,605 subjects at baseline enrollment. The TJEZ cohort will be followed up every 3 years, and a total of 4,695 subjects participated in the first follow-up survey which was conducted between 2016 and 2018, with a follow-up rate of $91.1 \%$. In the first follow-up survey, 3,204 cohort members were invited to undergo lipid remeasurements and genotyping; the 2,691 (84.0\%) respondents were broadly representative of the total cohort; 1,957 subjects had lipid measurements, and 1,655 individuals among them had genotyping results at baseline. After excluding subjects on lipid-lowering drugs, a subset of 1,621 subjects who had genotyping results at baseline as well as lipid measurements in the first follow-up survey, were included in the present study.

The study was approved by the Ethics and Human Subject Committee of Tongji Medical College, and written informed consent was obtained from all participants.

\section{Lipid Measurement}

Fasting serum lipid levels, including triglycerides, total cholesterol (TC), high-density lipoprotein cholesterol (HDL-C), low-density lipoprotein cholesterol (LDL-C), were measured by Hitachi automatic analyzer. Both the intra-assay and interassay variation coefficients of lipid levels were below $5 \%$. The non-high-density lipoprotein cholesterol (non-HDL-C) level was calculated as TC subtracting HDL-C. Additionally, the TC/HDL-C and LDL-C/HDL-C ratio were calculated. Changes 
in lipid levels during 3-year follow-up period were calculated by subtracting the lipids at follow-up from lipids at baseline.

\section{Measurement of Plasma Selenium Concentrations}

Plasma selenium concentrations were measured by inductively coupled plasma mass spectrometry (Agilent 7700 Series, Tokyo, Japan) in the Ministry of Education Key Laboratory of Environment and Health at Tongji Medical College of Huazhong University of Science \& Technology, as described previously (5). Prior to analysis, thawed samples ( $40 \mu \mathrm{L}$ plasma) were diluted with ultrapure water and digestive solution at a ratio of 1:19:20. For quality assurance, standard reference material ClinChek No. 8883 and No. 8884 human plasma controls for trace elements were analyzed in every 20 samples. The detection limit for plasma selenium was $0.024 \mu \mathrm{g} / \mathrm{L}$, and no samples had a level below this limit. Both intra-assay and inter-assay variation coefficients of plasma selenium were below $5 \%$.

\section{Genotyping}

Genomic DNA was isolated from the peripheral blood sample by kit (Tiangen biotech, Beijing, China). The sample DNA was amplified by a multiplex polymerase chain reaction for locus-specific single-base extension reaction. Genotyping of rs7579 was done by the MassArray system (Agena iPLEXassay, San Diego, United States). The sequences for the forward primer are "ACGTTGGATGTGACGCTGAAAGAATCAGGC," and the sequences for the reverse primer are "ACGTTGGAT GTGTGTCTAGACTAAATTGGG." Genotyping was successful in $99.3 \%(1,609$ of 1,621$)$ of the participants. The genotypes distribution of rs7579 was in Hardy-Weinberg equilibrium.

\section{Assessment of Covariates}

Basic information including sex, age, smoking status, alcohol drinking status, physical activity, educational level, and medical history (including use of lipid-lowering medication) was obtained via semi-structured questionnaires at baseline and was included as adjustment variables. Smoking status was grouped into two categories: current smoking ( $\geq 1$ cigarette/day over the past 6 months) and non-smoking. Alcohol drinking status was classified as current drinking ( $\geq 1$ time/week over the past 6 months) and non-drinking. Physical activity was defined as regular exercise for at least $60 \mathrm{~min}$ per week over the past 6 months. We classified educational level into three categories: none or elementary school, middle school, and high school or college. Anthropometric measurements were performed by trained staff. Body weight and standing height were measured in light indoor clothing and without shoes. Body mass index (BMI) was calculated by dividing the weight in kilograms by the square of the height in meters.

\section{Statistical Analysis}

Descriptive statistics were shown as means \pm standard deviations for continuous variables if normally distributed, medians (interquartile ranges) for continuous variables if not normally distributed, and frequencies (percentages) for categorical variables. Basic characteristics were compared across quartiles of plasma selenium by analysis of variance (normal distribution) or Kruskal-Wallis test (skewed distribution) for continuous variables, and $\chi^{2}$-test for categorical variables. Multivariable linear regression models were used and regression coefficients ( $95 \%$ CIs) were calculated to examine the associations of plasma selenium concentrations, as well as rs7579 polymorphisms, with 3-year changes in lipid levels. In multivariate models, we adjusted for respective baseline lipid (continuous) in model 1. In model 2, we further adjusted for sex (men/women), age (continuous), and BMI (continuous). In model 3, we further adjusted for current smoking status (yes/no), current drinking status (yes/no), physical activity (yes/no), and educational level (none or elementary school/middle school/high school or beyond). Due to right-skewed distribution, all lipids were ln-transformed and multiplied by 100 before analyses. Changes in lipid levels during 3-year follow-up period were calculated by subtracting the $100^{*} \ln$-transformed lipids at follow-up from $100^{*} \ln$-transformed lipids at baseline. Hence, regression coefficients in models can be interpreted as percentage difference in means (19).

To examine whether the associations between plasma selenium and 3-year changes in lipid levels were different across rs7579 genotypes, analyses of plasma selenium with 3-year lipid changes were stratified by rs7579 genotypes, and interactions between plasma selenium and rs7579 on 3-year lipid changes were identified through $1 \mathrm{df}$ multiplicative terms tests. Statistical power for the selenium $\times$ rs7579 interactions with lipid changes was calculated using QUANTO 1.2.4 (http://biostats.usc.edu/ Quanto.html). Assuming a marginal $R_{G}^{2}$ of 0.008 , a marginal $R_{E}^{2}$ of 0.01 , and a marginal $R_{G E}^{2}$ of 0.006 , our study had $88 \%$ power to detect an interaction for a lipid change.

All analyses were conducted using SAS 9.4 (SAS Instituted Inc, Cary, NC), and two-side $P<0.05$ were considered statistically significant.

\section{RESULTS}

General characteristics of participants are shown in Table 1. The 1,621 participants had a mean age of 57.86 years, and $65.3 \%$ were male. The median (interquartile range) concentration of plasma selenium was $91.68(81.55-104.92) \mu \mathrm{g} / \mathrm{L}$. At baseline, participants with higher plasma selenium concentrations were more likely to be men, current drinkers, and had higher levels of TC, LDL-C, and non-HDL-C.

After adjustment for sex, age, BMI, educational level, lifestyle factors, and respective baseline lipid, plasma selenium concentrations were positively associated with 3-year changes in triglycerides, TC, LDL-C, non-HDL-C, TC/HDL-C ratio, and LDL-C/HDL-C ratio (Table 2). Comparing the highest to the lowest quartiles of plasma selenium concentrations, 3-year changes in lipid levels were elevated by $8.25 \%$ (95\% CI: $1.54-$ $14.96 \%)$ for triglycerides $(P=0.016), 5.88 \%(3.13-8.63 \%)$ for TC $(P<0.001), 7.37 \%$ (3.07-11.67\%) for LDL-C $(P=0.0008), 6.44 \%$ (2.66-10.21\%) for non-HDL-C $(P=0.0009), 4.99 \%(0.62-9.36 \%)$ for TC/HDL-C ratio $(P=0.025)$, and $7.00 \%(1.55-12.46 \%)$ for LDL-C/HDL-C ratio $(P=0.012)$. The associations between 
TABLE 1 | Baseline characteristics of participants ${ }^{a}$.

\begin{tabular}{|c|c|c|c|c|c|c|}
\hline \multirow[t]{2}{*}{ Characteristics } & \multirow[t]{2}{*}{ Total } & \multicolumn{4}{|c|}{ Quartiles of plasma selenium concentrations ( $\mu \mathrm{g} / \mathrm{L})$} & \multirow[t]{2}{*}{$P$-value } \\
\hline & & Q1, $<81.56$ & Q2, 81.57-91.68 & Q3, 91.69-104.90 & $Q 4, \geq 104.91$ & \\
\hline No of participants & 1,621 & 406 & 405 & 405 & 405 & \\
\hline Men, $n(\%)$ & 1,058 (65.3) & $253(62.3)$ & $256(63.2)$ & $257(63.5)$ & $292(72.1)$ & 0.010 \\
\hline Age, years & $57.86(10.25)$ & $56.97(11.20)$ & $58.16(10.72)$ & $58.13(9.54)$ & $58.19(9.40)$ & 0.247 \\
\hline $\mathrm{BMl}, \mathrm{kg} / \mathrm{m}^{2}$ & $23.79(3.13)$ & $23.80(3.27)$ & $23.72(3.02)$ & $23.61(3.15)$ & $24.03(3.06)$ & 0.273 \\
\hline Triglycerides, mmol/L & $1.24(0.88,1.78)$ & $1.19(0.84,1.74)$ & $1.28(0.92,1.84)$ & $1.23(0.89,1.70)$ & $1.28(0.89,1.96)$ & 0.105 \\
\hline $\mathrm{TC}, \mathrm{mmol} / \mathrm{L}$ & $4.75(4.18,5.36)$ & $4.58(3.91,5.12)$ & $4.62(4.10,5.31)$ & $4.87(4.28,5.43)$ & $4.95(4.32,5.56)$ & $<0.001$ \\
\hline HDL-C, mmol/L & $1.32(1.10,1.58)$ & $1.27(1.05,1.54)$ & $1.33(1.09,1.57)$ & $1.35(1.14,1.59)$ & $1.34(1.11,1.61)$ & 0.008 \\
\hline LDL-C, mmol/L & $2.82(2.28,3.33)$ & $2.72(2.15,3.17)$ & $2.79(2.25,3.31)$ & $2.87(2.41,3.36)$ & $2.89(2.29,3.56)$ & $<0.001$ \\
\hline Non-HDL-C, mmol/L & $3.37(2.80,4.01)$ & $3.20(2.65,3.78)$ & $3.29(2.80,3.99)$ & $3.48(2.86,4.06)$ & $3.58(2.98,4.20)$ & $<0.001$ \\
\hline TC/HDL-C ratio & $3.56(2.96,4.36)$ & $3.51(2.87,4.37)$ & $3.57(2.94,4.30)$ & $3.51(2.94,4.29)$ & $3.66(3.05,4.49)$ & 0.154 \\
\hline LDL-C/HDL-C ratio & $2.12(1.63,2.72)$ & $2.10(1.63,2.72)$ & $2.11(1.64,2.72)$ & $2.08(1.59,2.71)$ & $2.21(1.71,2.75)$ & 0.549 \\
\hline Education level, $n$ (\%) & & & & & & 0.091 \\
\hline None or elementary school & $424(26.2)$ & $99(24.4)$ & 95 (23.5) & $105(25.9)$ & $125(30.9)$ & \\
\hline Middle school & $734(45.3)$ & $174(42.8)$ & $196(48.4)$ & $191(47.2)$ & $173(42.7)$ & \\
\hline High school or beyond & 463 (28.5) & $133(32.8)$ & $114(28.1)$ & 109 (26.9) & $107(26.4)$ & \\
\hline Current smoker, $n$ (\%) & 479 (29.5) & $120(29.6)$ & $122(30.1)$ & $116(28.6)$ & $121(29.9)$ & 0.970 \\
\hline Current drinker, $n(\%)$ & $480(29.6)$ & $99(24.4)$ & $109(26.9)$ & $120(29.6)$ & $152(37.5)$ & $<0.001$ \\
\hline Vigorous activity, n (\%) & 738 (45.5) & $180(44.3)$ & 198 (48.9) & $177(43.7)$ & $183(45.2)$ & 0.450 \\
\hline \multicolumn{7}{|l|}{ rs7579 genotypes, $n(\%)^{b}$} \\
\hline CC & $882(54.7)$ & $209(51.7)$ & $235(58.1)$ & $225(56.1)$ & $213(53.0)$ & 0.234 \\
\hline CT & $614(38.1)$ & $159(39.4)$ & $140(34.7)$ & $156(38.9)$ & 159 (39.5) & \\
\hline$\pi$ & $115(7.1)$ & $36(8.9)$ & $29(7.2)$ & $20(5.0)$ & $30(7.5)$ & \\
\hline Plasma selenium, $\mu \mathrm{g} / \mathrm{L}$ & $91.68(81.55,104.92)$ & $75.25(68.92,78.50)$ & $87.09(84.16,89.11)$ & $97.86(94.50,101.08)$ & $114.67(108.91,126.16)$ & $<0.001$ \\
\hline
\end{tabular}

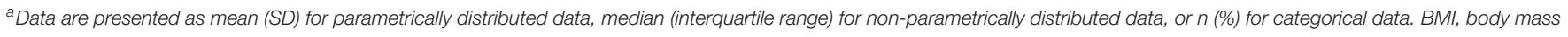
index; HDL-C, high-density lipoprotein cholesterol; LDL-C, low-density lipoprotein cholesterol; Non-HDL-C, non-high-density lipoprotein cholesterol; TC, total cholesterol.

${ }^{b}$ Genotyping of rs 7579 polymorphism was failed in 12 participants. Bold values indicates $P<0.05$.

rs7579 polymorphism and 3-year changes in lipids were showed in Table 3. After adjustment for sex, age, BMI, educational level, lifestyle factors, and respective baseline lipid, 3-year changes in lipid levels were elevated by $0.90 \%$ (95\% CI: $-8.41-10.21 \%)$ for triglycerides $(P=0.849),-0.47 \%(-4.32-3.39 \%)$ for TC $(P=$ $0.813), 0.67 \%(-4.85-6.20 \%)$ for HDL-C $(P=0.811),-2.67 \%$ $(-8.72-3.39 \%)$ for LDL-C $(P=0.388), 0.47 \%(-4.85-5.79 \%)$ for non-HDL-C $(P=0.861),-1.53 \%(-7.70-4.63 \%)$ for TC/HDL-C ratio $(P=0.626)$, and $-3.20 \%(-10.89-4.49 \%)$ for LDL-C/HDL$\mathrm{C}$ ratio $(P=0.415)$, when comparing the TT genotype with the TT genotype carriers.

Analyses of plasma selenium with 3-year changes in lipid levels were stratified by rs7579 genotypes (Figure 1). In stratified analyses, positive associations between plasma selenium concentrations and 3-year changes in triglycerides, TC, LDL-C, non-HDL-C, TC/HDL-C ratio, and LDL-C/HDL-C ratio were observed among $\mathrm{CC}$ genotype carriers, but negative associations between plasma selenium and TC/HDL-C ratio, and LDL$\mathrm{C} / \mathrm{HDL}-\mathrm{C}$ ratio were observed among TT genotype carriers. Among rs7579 CC genotype carriers, comparing the highest to the lowest quartiles of plasma selenium concentrations, 3year changes in lipid levels were elevated by $11.54 \%(2.29-$ $20.79 \%)$ for triglycerides $(P=0.015), 9.39 \%(5.53-13.24 \%)$ for TC $(P<0.001),-0.41 \%(-5.94-5.11 \%)$ for HDL-C $(P=$ $0.884), 11.66 \%(5.72-17.61 \%)$ for LDL-C $(P=0.0001), 10.91 \%$
(5.64-16.18\%) for non-HDL-C $(P<0.001), 9.44 \%$ (3.36-15.51\%) for TC/HDL-C ratio $(P=0.002)$, and $12.35 \%(5.19-19.51 \%)$ for $\mathrm{LDL}-\mathrm{C} / \mathrm{HDL}-\mathrm{C}$ ratio $(P=0.0007)$. Among TT genotype carriers, comparing the highest to the lowest quartiles of plasma selenium concentrations, 3-year changes in lipid levels were elevated by $26.45 \%(-3.43-56.33 \%)$ for triglycerides $(P=0.082)$, $6.23 \%(-4.65-17.10 \%)$ for TC $(P=0.259), 15.41 \%(-6.15-$ $36.97 \%)$ for HDL-C $(P=0.159), 8.56 \%(-14.93-32.06 \%)$ for LDL-C $(P=0.471), 4.06 \%(-11.20-19.31 \%)$ for non-HDL-C $(P$ $=0.599),-5.45 \%(-32.65-21.76 \%)$ for $\mathrm{TC} / \mathrm{HDL}-\mathrm{C}$ ratio $(P=$ $0.692)$, and $-3.88 \%(-41.99-34.24 \%)$ for LDL-C/HDL-C ratio $(P=0.840)$. The interactions between plasma selenium and rs7579 polymorphism on 3-year changes in TC ( $P$ for interaction $=0.007)$, LDL-C $(P$ for interaction $=0.034)$, non-HDL-C $(P$ for interaction $=0.005)$, TC/HDL-C ratio $(P$ for interaction $=$ $0.034)$, and LDL-C/HDL-C ratio $(P$ for interaction $=0.048)$ were statistically significant.

\section{DISCUSSION}

In this study, plasma selenium was positively associated with 3-year changes in triglycerides, TC, LDL-C, non-HDL$\mathrm{C}$, TC/HDL-C ratio, and LDL-C/HDL-C ratio. Moreover, the positive associations between plasma selenium and 3year changes in lipid levels were only significant in rs7579 
TABLE 2 | Mean \% difference (95\% Cl) in 3-year changes in lipid levels according to quartiles of plasma selenium concentrations ${ }^{a}$.

\begin{tabular}{|c|c|c|c|c|c|}
\hline \multirow{2}{*}{$\begin{array}{l}\text { Mean \% (95\% Cl) difference in } \\
\text { 3-year changes in lipid levels }\end{array}$} & \multicolumn{4}{|c|}{ Quartiles of plasma selenium concentrations $(\mu \mathrm{g} / \mathrm{L})$} & \multirow[t]{2}{*}{$P$-trend } \\
\hline & Q1, <81.56 & Q2, 81.57-91.68 & Q3, 91.69-104.90 & $Q 4, \geq 104.91$ & \\
\hline \multicolumn{6}{|l|}{ Triglycerides } \\
\hline Model 1 & 0.00 (referent) & $1.57(-5.25,8.39)$ & $1.78(-5.00,8.55)$ & $6.87(0.10,13.64)^{\star}$ & 0.056 \\
\hline Model 2 & 0.00 (referent) & $2.85(-3.87,9.57)$ & $2.73(-3.93,9.40)$ & $7.42(0.75,14.09)^{\star}$ & 0.040 \\
\hline Model 3 & 0.00 (referent) & $2.90(-3.82,9.62)$ & $3.13(-3.55,9.81)$ & $8.25(1.54,14.96)^{\star}$ & 0.021 \\
\hline \multicolumn{6}{|l|}{ TC } \\
\hline Model 1 & 0.00 (referent) & $2.70(-0.14,5.53)$ & $4.08(1.26,6.91)^{\star}$ & $5.40(2.58,8.22)^{\star}$ & $<0.001$ \\
\hline Model 2 & 0.00 (referent) & $3.31(0.56,6.05)^{\star}$ & $4.28(1.55,7.02)^{\star}$ & $5.87(3.14,8.61)^{\star}$ & $<0.001$ \\
\hline Model 3 & 0.00 (referent) & $3.23(0.48,5.98)^{\star}$ & $4.26(1.52,7.00)^{\star}$ & $5.88(3.13,8.63)^{\star}$ & $<0.001$ \\
\hline \multicolumn{6}{|l|}{ HDL-C } \\
\hline Model 1 & 0.00 (referent) & $1.09(-3.10,5.28)$ & $-1.18(-5.36,3.00)$ & $0.17(-4.00,4.33)$ & 0.797 \\
\hline Model 2 & 0.00 (referent) & $2.21(-1.74,6.16)$ & $-0.95(-4.88,2.98)$ & $0.97(-2.96,4.89)$ & 0.970 \\
\hline Model 3 & 0.00 (referent) & $2.14(-1.80,6.08)$ & $-1.30(-5.22,2.62)$ & $0.40(-3.52,4.32)$ & 0.729 \\
\hline \multicolumn{6}{|l|}{ LDL-C } \\
\hline Model 1 & 0.00 (referent) & $3.58(-0.76,7.92)$ & $4.89(0.56,9.21)^{\star}$ & $6.65(2.34,10.96)^{\star}$ & 0.002 \\
\hline Model 2 & 0.00 (referent) & $4.27(-0.03,8.57)$ & $5.30(1.02,9.59)^{\star}$ & $7.27(2.99,11.55)^{\star}$ & 0.001 \\
\hline Model 3 & 0.00 (referent) & $4.27(-0.04,8.58)$ & $5.39(1.10,9.69)^{\star}$ & $7.37(3.07,11.67)^{\star}$ & $<0.001$ \\
\hline \multicolumn{6}{|l|}{ Non-HDL-C } \\
\hline Model 1 & 0.00 (referent) & $3.20(-0.65,7.04)$ & $4.77(0.94,8.61)^{\star}$ & $5.77(1.94,9.59)^{\star}$ & 0.002 \\
\hline Model 2 & 0.00 (referent) & $3.99(0.22,7.76)^{\star}$ & $5.21(1.45,8.97)^{\star}$ & $6.27(2.51,10.02)^{\star}$ & 0.001 \\
\hline Model 3 & 0.00 (referent) & $3.96(0.18,7.74)^{\star}$ & $5.30(1.53,9.07)^{\star}$ & $6.44(2.66,10.21)^{\star}$ & $<0.001$ \\
\hline \multicolumn{6}{|l|}{ TC/HDL-C ratio } \\
\hline Model 1 & 0.00 (referent) & $1.68(-2.76,6.12)$ & $5.00(0.59,9.41)^{\star}$ & $4.60(0.20,9.00)^{\star}$ & 0.016 \\
\hline Model 2 & 0.00 (referent) & $1.29(-3.11,5.69)$ & $4.90(0.53,9.27)^{\star}$ & $4.31(-0.05,8.68)$ & 0.019 \\
\hline Model 3 & 0.00 (referent) & $1.33(-3.07,5.72)$ & $5.32(0.95,9.69)^{\star}$ & $4.99(0.62,9.36)^{\star}$ & 0.007 \\
\hline \multicolumn{6}{|l|}{ LDL-C/HDL-C ratio } \\
\hline Model 1 & 0.00 (referent) & $2.73(-2.81,8.28)$ & $6.62(1.11,12.13)^{\star}$ & $6.71(1.22,12.21)^{\star}$ & 0.007 \\
\hline Model 2 & 0.00 (referent) & $2.05(-3.43,7.53)$ & $6.38(0.93,11.82)$ & $6.35(0.91,11.79)^{\star}$ & 0.008 \\
\hline Model 3 & 0.00 (referent) & $2.16(-3.32,7.65)$ & $6.85(1.41,12.30)^{*}$ & $7.00(1.55,12.46)^{\star}$ & 0.004 \\
\hline
\end{tabular}

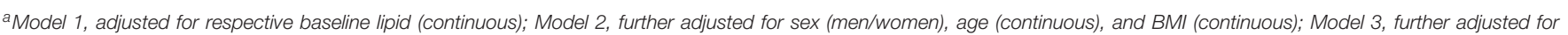

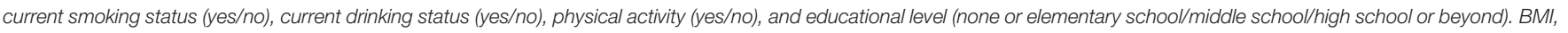
body mass index; HDL-C, high-density lipoprotein cholesterol; LDL-C, low-density lipoprotein cholesterol; Non-HDL-C, non-high-density lipoprotein cholesterol; TC, total cholesterol. ${ }^{\star} P<0.05$. Bold values indicates $P<0.05$.

CC genotype carriers, while non-significant associations were observed in $\mathrm{T}$ allele carriers.

The selenium status among people varies by regions and mainly depends on its dietary intake $(20,21)$. The intakes are high in America, Japan, and Canada, and much lower in the majority of Eastern Europe. China has both high-selenium and low-selenium areas (20). Although the optimal selenium status remains controversial, findings from some studies suggested the plasma selenium concentration around $90 \mu \mathrm{g} / \mathrm{L}$ to be the optimal value, which maximizes the plasma SELENOP level (20). The median concentration of plasma selenium was $91.68 \mu \mathrm{g} / \mathrm{L}$ in our participants, which was considered as selenium-sufficient.

Our findings of positive associations between plasma selenium and changes in triglycerides, TC, LDL-C, non-HDL-C, TC/HDL$\mathrm{C}$ ratio, and $\mathrm{LDL}-\mathrm{C} / \mathrm{HDL}-\mathrm{C}$ ratio are consistent with those from cross-sectional studies conducted among subjects with a wide-range of selenium status, which also demonstrated strong, positive associations between selenium status and lipid levels (3, 4, 21-24). For example, in the third National Health and Nutrition Examination Survey (NHANES III), which included a representative American population (median blood selenium $199.88 \mu \mathrm{g} / \mathrm{L}$ ), increased serum selenium was significantly associated with higher lipid levels (21). Also, another crosssectional study conducted among a selenium-excess Chinese population (mean serum selenium $120 \mu \mathrm{g} / \mathrm{L}$ ) revealed significant positive associations of plasma selenium with triglycerides, TC, and LDL-C (3). In addition, several cross-sectional studies conducted among selenium-deficient individuals (mean serum selenium $79.5 \mu \mathrm{g} / \mathrm{L}$ ) also revealed that higher plasma selenium was associated with adverse lipid levels (7, 25). Limited longitudinal studies have explored relationships between baseline blood selenium and changes in lipid levels, and findings from them indicated non-significant or negative associations between selenium status and changes in lipid levels $(6,8)$. It seems likely 
TABLE 3 | Mean \% difference (95\% Cl) in 3-year changes in lipid levels according to rs7579 genotypes ${ }^{\mathrm{a}}$.

\begin{tabular}{|c|c|c|c|c|}
\hline \multirow{2}{*}{$\begin{array}{l}\text { Mean } \%(95 \% \mathrm{Cl}) \text { difference in 3-year } \\
\text { changes in lipid levels }\end{array}$} & \multicolumn{3}{|c|}{ rs7579 genotypes } & \multirow[t]{2}{*}{$P$-trend } \\
\hline & CC & CT & TT & \\
\hline \multicolumn{5}{|l|}{ Triglycerides } \\
\hline Model 1 & 0.00 (referent) & $-2.58(-7.61,2.45)$ & $2.90(-6.55,12.35)$ & 0.835 \\
\hline Model 2 & 0.00 (referent) & $-2.92(-7.87,2.03)$ & $0.99(-8.32,10.30)$ & 0.573 \\
\hline Model 3 & 0.00 (referent) & $-3.13(-8.10,1.83)$ & $0.90(-8.41,10.21)$ & 0.532 \\
\hline \multicolumn{5}{|l|}{ TC } \\
\hline Model 1 & 0.00 (referent) & $-0.99(-3.11,1.12)$ & $0.46(-3.52,4.43)$ & 0.683 \\
\hline Model 2 & 0.00 (referent) & $-1.33(-3.38,0.72)$ & $-0.41(-4.26,3.45)$ & 0.360 \\
\hline Model 3 & 0.00 (referent) & $-1.23(-3.28,0.83)$ & $-0.47(-4.32,3.39)$ & 0.383 \\
\hline \multicolumn{5}{|l|}{ HDL-C } \\
\hline Model 1 & 0.00 (referent) & $1.86(-1.25,4.97)$ & $2.25(-3.63,8.12)$ & 0.223 \\
\hline Model 2 & 0.00 (referent) & $1.02(-1.91,3.95)$ & $0.78(-4.76,6.33)$ & 0.547 \\
\hline Model 3 & 0.00 (referent) & $1.36(-1.57,4.29)$ & $0.67(-4.85,6.20)$ & 0.473 \\
\hline \multicolumn{5}{|l|}{ LDL-C } \\
\hline Model 1 & 0.00 (referent) & $0.81(-2.42,4.04)$ & $-1.71(-7.81,4.39)$ & 0.948 \\
\hline Model 2 & 0.00 (referent) & $0.49(-2.70,3.69)$ & $-2.68(-8.72,3.36)$ & 0.691 \\
\hline Model 3 & 0.00 (referent) & $0.53(-2.68,3.74)$ & $-2.67(-8.72,3.39)$ & 0.704 \\
\hline \multicolumn{5}{|l|}{ Non-HDL-C } \\
\hline Model 1 & 0.00 (referent) & $-1.60(-4.45,1.26)$ & $1.62(-3.80,7.03)$ & 0.779 \\
\hline Model 2 & 0.00 (referent) & $-1.97(-4.77,0.83)$ & $0.51(-4.80,5.82)$ & 0.470 \\
\hline Model 3 & 0.00 (referent) & $-1.95(-4.76,0.86)$ & $0.47(-4.85,5.79)$ & 0.473 \\
\hline \multicolumn{5}{|l|}{ TC/HDL-C ratio } \\
\hline Model 1 & 0.00 (referent) & $-3.03(-6.33,0.26)$ & $-1.88(-8.10,4.35)$ & 0.134 \\
\hline Model 2 & 0.00 (referent) & $-2.61(-5.88,0.65)$ & $-1.56(-7.74,4.61)$ & 0.196 \\
\hline Model 3 & 0.00 (referent) & $-2.89(-6.16,0.38)$ & $-1.53(-7.70,4.63)$ & 0.165 \\
\hline \multicolumn{5}{|l|}{ LDL-C/HDL-C ratio } \\
\hline Model 1 & 0.00 (referent) & $-1.12(-5.23,3.00)$ & $-3.99(-11.77,3.79)$ & 0.318 \\
\hline Model 2 & 0.00 (referent) & $-0.54(-4.61,3.52)$ & $-3.29(-10.99,4.40)$ & 0.471 \\
\hline Model 3 & 0.00 (referent) & $-0.84(-4.92,3.24)$ & $-3.20(-10.89,4.49)$ & 0.427 \\
\hline
\end{tabular}

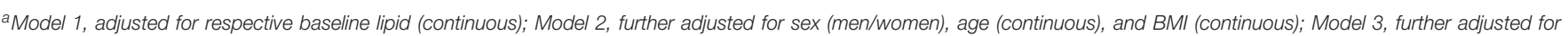

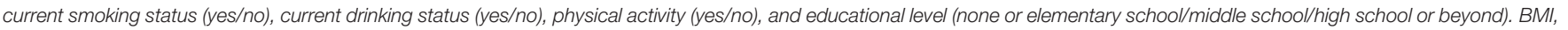
body mass index; HDL-C, high-density lipoprotein cholesterol; LDL-C, low-density lipoprotein cholesterol; Non-HDL-C, non-high-density lipoprotein cholesterol; TC, total cholesterol.

that there are some other unmeasured factors might confound the longitudinal association between selenium status and lipid levels. Additionally, in view of genetic factors account for nearly $30 \%$ of lipids, such contradictory findings might be partly due to difference in genetic background of participants between studies. More longitudinal studies examining associations of selenium status with changes in lipid levels are warranted to further verify our findings.

Although exact mechanisms underlying the adverse effects of increased selenium on lipid changes among seleniumsufficient or selenium-excess subjects are not clearly understood, several potential mechanisms might be involved, including endoplasmic reticulum stress, mitochondrial dysfunction, and changed miRNA expression. Firstly, excess selenium has been suggested to induce endoplasmic reticulum stress (26). Under endoplasmic reticulum stress, the expression of several key factors in the pathway of de novo lipogenesis was upregulated in the liver, including the sterol regulatory element binding protein 1c (SREBP1c) and the fatty acid synthase (FASN) (27). Secondly, high selenium might be involved in abnormal lipid metabolism through disturbing mitochondrial function. Romero et al. revealed that high selenium exposures could induce mitochondrial dysfunction by increasing the generation of reactive oxygen species (28). Mitochondrial dysfunction could disturb lipid metabolism through several mechanisms, including leading to endoplasmic reticulum stress, impairing autophagy, and then increasing lipogenesis (29, 30). And lastly, Guo et al. found blood selenium was positively associated with the expression of miR-122-5p (31), which has been implicated as a key regulator of cholesterol and fatty-acid metabolism in the liver (32). Nevertheless, more mechanistic studies are needed to further illustrate potential mechanisms through which high selenium plays a role in adverse changes in lipid levels.

Our study also revealed significant interactions between plasma selenium and rs7579 polymorphism on changes in lipid levels, which helped to explain the inconsistent findings between 

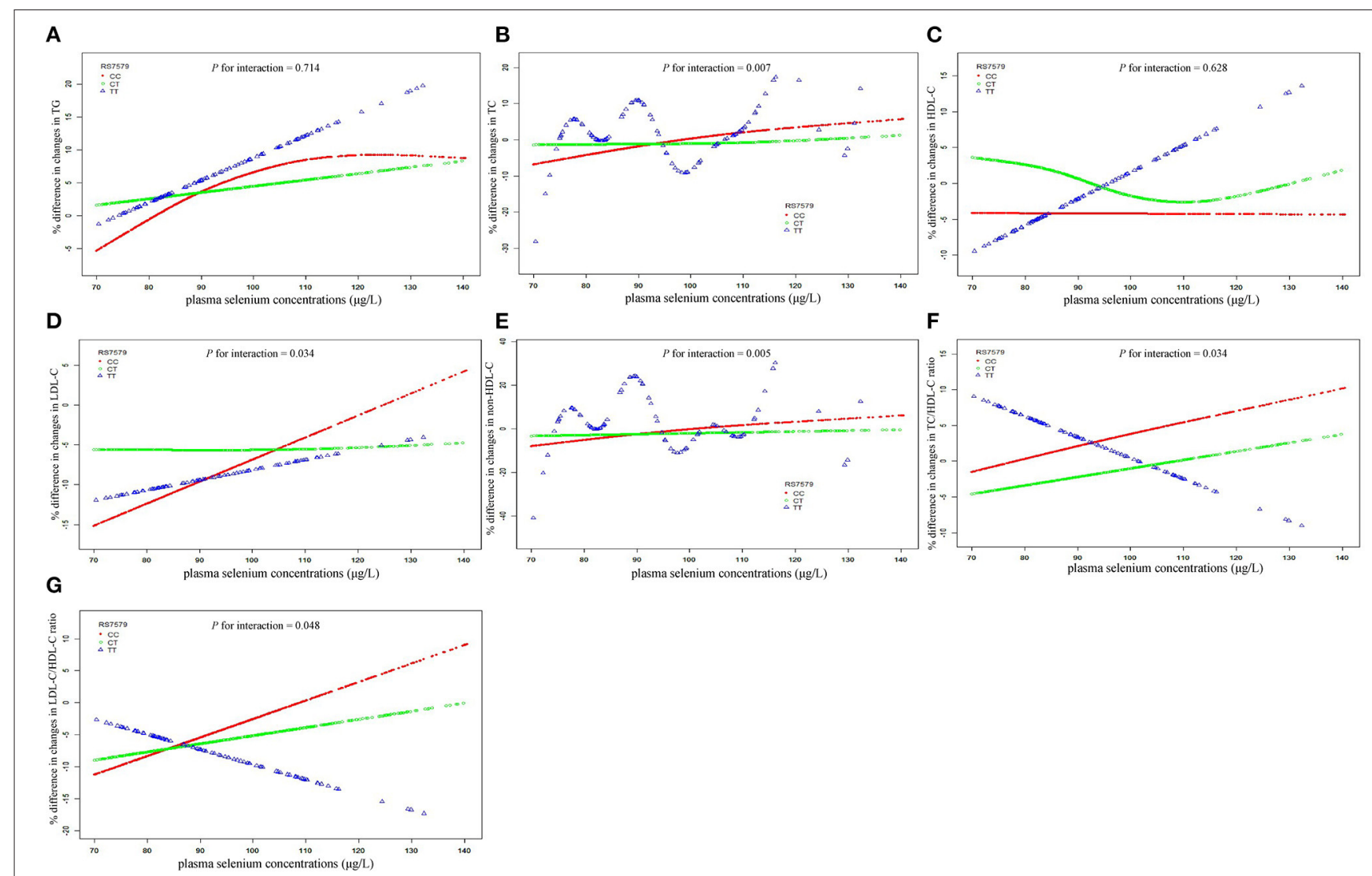

FIGURE 1 | Associations of plasma selenium concentrations with 3-year changes in lipids stratified by rs7579 genotypes. X bars represent plasma selenium concentrations. Y bars represent mean \% difference in 3-year changes in triglycerides for (A), TC for (B), HDL-C for (C), LDL-C for (D), non-HDL-C for (E), TC/HDL-C ratio for (F), and LDL-C/HDL-C ratio for (G). Multivariable analyses were adjusted for sex (men/women), age (continuous), BMI (continuous), current smoking status (yes/no), current drinking status (yes/no), physical activity (yes/no), educational level (none or elementary school/middle school/high school or beyond), and respective baseline lipid (continuous). BMI, body mass index; HDL-C, high-density lipoprotein cholesterol; LDL-C, low-density lipoprotein cholesterol; Non-HDL-C,

non-high-density lipoprotein cholesterol; TC, total cholesterol.

studies and suggested that such inconsistency might be partly due to the selenium $\times$ rs7579 polymorphism interactions. We showed that the impact of baseline selenium on changes in lipid levels depended on genotype at rs7579. Notably, the adverse effect of high selenium on changes in lipid levels was only significant among rs7579 CC genotype carriers, but non-significant among rs7579 T allele carriers. Although such an interaction has not been reported before, there are studies that corroborate our findings. Findings from an intervention trial suggested that the changes in cholesterol levels in response to Brazil nut supplementation were greater among rs7579 CC genotype than $\mathrm{T}$ allele carriers (18), which is in line with the currently observed differential associations of plasma selenium with lipid changes across rs7579 genotypes. Furthermore, it has been suggested that both the mRNA expression and plasma levels of SELENOP were significantly lower among rs7579 CC than T allele carriers (16, 17), and decreased plasma SELENOP was related with increased risks of several dyslipidemia-related diseases, including metabolic syndrome, cerebrovascular events, and cardiovascular disease (33-35). Our study also revealed non-significant associations between rs7579 polymorphism and changes in lipid levels, which is consistent with those from previous studies.

Our study has several strengths. First, to our knowledge, this is the first population-study systematically exploring the interactions between plasma selenium and rs7579 polymorphism with changes in lipid levels. Second, the prospective design allowed us to clarify the temporal sequence of the relationships between selenium and lipid levels. Third, we assess selenium status using plasma selenium concentration instead of dietary intake of selenium to avoid possible bias through dietary survey.

Several limitations should also be acknowledged. First, plasma selenium concentrations were measured only once, and measurement errors and exposure misclassification were inevitable. However, such errors were likely to be non-differential and thus would be more likely to induce the attenuation of the observed associations. Second, we only measured total plasma selenium levels rather than different chemical forms of selenium. Given that different chemical forms of selenium might have different properties and effects on human health, more detailed analyses of different chemical forms of selenium are 
needed to better understand the relationships between selenium and lipid changes. Also, we did not have measurement of plasma SELENOP levels, which precluded us from exploring the associations of rs7579 polymorphism as well as lipid changes with plasma SELENOP levels. Third, our study was conducted among a selenium-appropriate population. Thus, the generalizability of our findings to people with different selenium statuses remains unclear, and further studies are needed to determine the generalizability of our findings. Fourth, the study population was limited to a Chinese population, which also limits the generalizability of our findings. Finally, no information on health status (e.g., obese, diabetic) was collected in our population, which should be taken into account in future study. In addition, although multiple potential confounders have been adjusted, we could not rule out the possibility of residual confounding by other unknown or unmeasured factors.

In conclusion, our findings demonstrated that, in a seleniumappropriate population, plasma selenium was associated with lipid changes differentially across rs7579 genotypes, and higher selenium seemed to be associated with adverse changes in lipid levels among rs7579 CC genotype carriers, but not among $\mathrm{T}$ allele carriers. These findings warn the widespread use of seleniumfortified foods or selenium supplements, especially among selected study populations, such as rs7579 CC genotype carriers.

\section{DATA AVAILABILITY STATEMENT}

The raw data supporting the conclusions of this article will be made available by the authors, without undue reservation.

\section{ETHICS STATEMENT}

The studies involving human participants were reviewed and approved by the Ethics and Human Subject Committee of Tongji

\section{REFERENCES}

1. Rayman MP. Selenium and human health. Lancet. (2012) 379:125668. doi: 10.1016/S0140-6736(11)61452-9

2. Bodnar M, Szczyglowska M, Konieczka P, Namiesnik J. Methods of selenium supplementation: bioavailability and determination of selenium compounds. Crit Rev Food Sci Nutr. (2016) 56:36-55. doi: 10.1080/10408398.2012.709550

3. Ju W, Ji M, Li X, Li Z, Wu G, Fu X, et al. Relationship between higher serum selenium level and adverse blood lipid profile. Clin Nutr. (2018) 37:1512-17. doi: 10.1016/j.clnu.2017.08.025

4. Liu A, Xu P, Gong C, Zhu Y, Zhang H, Nie W, et al. High serum concentration of selenium, but not calcium, cobalt, copper, iron, and magnesium, increased the risk of both hyperglycemia and dyslipidemia in adults: a health examination center based cross-sectional study. J Trace Elem Med Biol. (2020) 59:126470. doi: 10.1016/j.jtemb.2020.1 26470

5. Zhou L, Luo C, Yin J, Zhu Y, Li P, Chen S, et al. Diverse associations of plasma selenium concentrations and SELENOP gene polymorphism with metabolic syndrome and its components. Oxid Med Cell Longev. (2020) 2020:5343014. doi: 10.1155/2020/5343014

6. Stranges S, Galletti F, Farinaro E, D'Elia L, Russo O, Iacone R, et al. Associations of selenium status with cardiometabolic risk factors: an 8-year follow-up analysis of the Olivetti Heart study. Atherosclerosis. (2011) 217:2748. doi: 10.1016/j.atherosclerosis.2011.03.027
Medical College. The patients/participants provided their written informed consent to participate in this study.

\section{AUTHOR CONTRIBUTIONS}

LZ contributed to the statistical analysis, interpretation of the results, and wrote the manuscript. LZ, XLia, MX, JY, and YH contributed to the acquisition of data and researched the data. $\mathrm{XLi}, \mathrm{ZS}$, and LC contributed to the study design. YZ contributed to the discussion of the project. LZ, XLia, MX, JY, YH, XLi, ZS, LC, YZ, CL, and LL reviewed and edited the manuscript. LL and CL are the guarantors of this work and as such, have full access to all the data in the study and take responsibility for the integrity of the data and the accuracy of the data analysis. All authors contributed to the article and approved the submitted version.

\section{FUNDING}

This work was supported by the Major International (Regional) Joint Research Project (NSFC 81820108027), the National Key Research and Development Program of China (2020YFC2006300), the National Natural Science Foundation of China (81803226), and the Angel Nutrition Research Fund (AF2019003). The funding sources had no role in the design and conduct of the study, collection, management, analysis, and interpretation of the data, preparation, review, or approval of the manuscript, and decision to submit the manuscript for publication.

\section{ACKNOWLEDGMENTS}

The authors thank all those who volunteered to take part in this research.
7. Stranges S, Laclaustra M, Ji C, Cappuccio FP, Navas-Acien A, Ordovas JM, et al. Higher selenium status is associated with adverse blood lipid profile in British adults. J Nutr. (2010) 140:81-7. doi: 10.3945/jn.109.111252

8. Stranges S, Tabak AG, Guallar E, Rayman MP, Akbaraly TN, Laclaustra $M$, et al. Selenium status and blood lipids: the cardiovascular risk in Young Finns study. J Intern Med. (2011) 270:469-77. doi: 10.1111/j.1365-2796.2011.02398.x

9. Teslovich TM, Musunuru K, Smith AV, Edmondson AC, Stylianou IM, Koseki $\mathrm{M}$, et al. Biological, clinical and population relevance of 95 loci for blood lipids. Nature. (2010) 466:707-13. doi: 10.1038/nature09270

10. Krauss RM. What can the genome tell us about LDL cholesterol? Lancet. (2008) 371:450-2. doi: 10.1016/S0140-6736(08)60213-5

11. Thomson CD, Chisholm A, McLachlan SK, Campbell JM. Brazil nuts: an effective way to improve selenium status. Am J Clin Nutr. (2008) 87:37984. doi: 10.1093/ajen/87.2.379

12. Hill KE, Zhou J, McMahan WJ, Motley AK, Atkins JF, Gesteland RF, et al. Deletion of selenoprotein $\mathrm{P}$ alters distribution of selenium in the mouse. J Biol Chem. (2003) 278:13640-6. doi: 10.1074/jbc.M300755200

13. Schomburg L, Schweizer U, Holtmann B, Flohe L, Sendtner M, Kohrle J. Gene disruption discloses role of selenoprotein $\mathrm{P}$ in selenium delivery to target tissues. Biochem J. (2003) 370 (Pt 2):397-402. doi: 10.1042/bj20021853

14. Short SP, Pilat JM, Williams CS. Roles for selenium and selenoprotein P in the development, progression, and prevention of intestinal disease. Free Radic Biol Med. (2018) 127:26-35. doi: 10.1016/j.freeradbiomed.2018.05.066 
15. Short SP, Pilat JM, Barrett CW, Reddy VK, Haberman Y, Hendren JR, et al. Colonic epithelial-derived selenoprotein $\mathrm{P}$ is the source for antioxidantmediated protection in colitis-associated cancer. Gastroenterology. (2021) 160:5:1694-708.e3. doi: 10.1053/j.gastro.2020.12.059

16. Donadio JLS, Rogero MM, Cockell S, Hesketh J, Cozzolino SMF. Influence of genetic cariations in selenoprotein genes on the pattern of gene expression after supplementation with Brazil nuts. Nutrients. (2017) 9:7. doi: 10.3390/nu9070739

17. Donadio JLS, Rogero MM, Guerra-Shinohara EM, Barbosa F Jr, Desmarchelier C, Borel P, et al. Genetic variants in selenoprotein genes modulate biomarkers of selenium status in response to Brazil nut supplementation (the SU.BRA.NUT study). Clin Nutr. (2019) 38:539-48. doi: 10.1016/j.clnu.2018.03.011

18. Donadio JLS, Rogero MM, Guerra-Shinohara EM, Desmarchelier C, Borel P, Cozzolino SMF. SEPP1 polymorphisms modulate serum glucose and lipid response to Brazil nut supplementation. Eur J Nutr. (2018) 57:187382. doi: 10.1007/s00394-017-1470-7

19. Pinto Pereira SM, Power C. Life course body mass index, birthweight and lipid levels in mid-adulthood: a nationwide birth cohort study. Eur Heart J. (2013) 34:1215-24. doi: 10.1093/eurheartj/ehs333

20. Xia Y, Hill KE, Li P, Xu J, Zhou D, Motley AK, et al. Optimization of selenoprotein $\mathrm{P}$ and other plasma selenium biomarkers for the assessment of the selenium nutritional requirement: a placebo-controlled, double-blind study of selenomethionine supplementation in selenium-deficient Chinese subjects. Am J Clin Nutr. (2010) 92:525-31. doi: 10.3945/ajcn.2010.29642

21. Christensen K, Werner M, Malecki K. Serum selenium and lipid levels: associations observed in the National Health and Nutrition Examination Survey (NHANES) 2011-2012. Environ Res. (2015) 140:7684. doi: 10.1016/j.envres.2015.03.020

22. Fang $\mathrm{H}, \mathrm{He} \mathrm{X}, \mathrm{Wu} \mathrm{Y}$, Chen $\mathrm{S}$, Zhang $\mathrm{M}$, Pan $\mathrm{F}$, et al. Association between selenium level in blood and glycolipid metabolism in residents of Enshi prefecture, China. Biol Trace Elem Res. (2021) 199:7:245666. doi: 10.1007/s12011-020-02372-9

23. Huang YQ, Shen G, Lo K, Huang JY, Liu L, Chen CL, et al. Association of circulating selenium concentration with dyslipidemia: results from the NHANES. J Trace Elem Med Biol. (2020) 58:126438. doi: 10.1016/j.jtemb.2019.126438

24. Loomba R, Filippini T, Chawla R, Chaudhary R, Cilloni S, Datt $\mathrm{C}$, et al. Exposure to a high selenium environment in Punjab, India: effects on blood chemistry. Sci Total Environ. (2020) 716:135347. doi: 10.1016/j.scitotenv.2019.135347

25. Yang KC, Lee LT, Lee YS, Huang HY, Chen CY, Huang KC. Serum selenium concentration is associated with metabolic factors in the elderly: a crosssectional study. Nutr Metab. (2010) 7:38. doi: 10.1186/1743-7075-7-38

26. Zachariah M, Maamoun H, Milano L, Rayman MP, Meira LB, Agouni A. Endoplasmic reticulum stress and oxidative stress drive endothelial dysfunction induced by high selenium. J Cell Physiol. (2021) 236:434859. doi: 10.1002/jcp.30175

27. Fu S, Watkins SM, Hotamisligil GS. The role of endoplasmic reticulum in hepatic lipid homeostasis and stress signaling. Cell Metab. (2012) 15:62334. doi: 10.1016/j.cmet.2012.03.007
28. Zhuang Y, Li L, Feng L, Wang S, Su H, Liu H, et al. Mitochondriontargeted selenium nanoparticles enhance reactive oxygen species-mediated cell death. Nanoscale. (2020) 12:3:1389-96. doi: 10.1039/C9NR0 $9039 \mathrm{H}$

29. Biczo G, Vegh ET, Shalbueva N, Mareninova OA, Elperin J, Lotshaw E, et al. Mitochondrial dysfunction, through impaired autophagy, leads to endoplasmic reticulum stress, deregulated lipid metabolism, and pancreatitis in animal models. Gastroenterology. (2018) 154:3:689-703. doi: 10.1053/j.gastro.2017.10.012

30. Peng KY, Watt MJ, Rensen S, Greve JW, Huynh K, Jayawardana KS, et al. Mitochondrial dysfunction-related lipid changes occur in nonalcoholic fatty liver disease progression. J Lipid Res. (2018) 59:10:197786. doi: 10.1194/jlr.M085613

31. Guo X, Yang Q, Zhang W, Chen Y, Ren J, Gao A. Associations of blood levels of trace elements and heavy metals with metabolic syndrome in Chinese male adults with microRNA as mediators involved. Environ Pollut. (2019) 248:66-73. doi: 10.1016/j.envpol.2019.02.015

32. Esau C, Davis S, Murray SF, Yu XX, Pandey SK, Pear M, et al. miR122 regulation of lipid metabolism revealed by in vivo antisense targeting. Cell Metab. (2006) 3:2:87-98. doi: 10.1016/j.cmet.2006. 01.005

33. Gharipour M, Sadeghi M, Salehi M, Behmanesh M, Khosravi E, Dianatkhah $\mathrm{M}$, et al. Association of expression of selenoprotein $\mathrm{P}$ in mRNA and protein levels with metabolic syndrome in subjects with cardiovascular disease: results of the selenegene study. J Gene Med. (2017) 19:3. doi: 10.1002/ jgm. 2945

34. Koyama H, Abdulah R, Ohkubo T, Imai Y, Satoh H, Nagai K. Depressed serum selenoprotein P: possible new predicator of increased risk for cerebrovascular events. Nut Res. (2009) 29:2:94-9. doi: 10.1016/j.nutres.2009.01.002

35. Schomburg L, Orho-Melander M, Struck J, Bergmann A, Melander O. Selenoprotein-P deficiency predicts cardiovascular disease and death. Nutrients. (2019) 11:8. doi: 10.3390/nu11081852

Conflict of Interest: The authors declare that the research was conducted in the absence of any commercial or financial relationships that could be construed as a potential conflict of interest.

Publisher's Note: All claims expressed in this article are solely those of the authors and do not necessarily represent those of their affiliated organizations, or those of the publisher, the editors and the reviewers. Any product that may be evaluated in this article, or claim that may be made by its manufacturer, is not guaranteed or endorsed by the publisher.

Copyright (c) 2021 Zhou, Liang, Xie, Yin, Huang, Li, Shan, Chen, Zhang, Luo and Liu. This is an open-access article distributed under the terms of the Creative Commons Attribution License (CC BY). The use, distribution or reproduction in other forums is permitted, provided the original author(s) and the copyright owner(s) are credited and that the original publication in this journal is cited, in accordance with accepted academic practice. No use, distribution or reproduction is permitted which does not comply with these terms. 